\title{
TIERED TREES, WEIGHTS, AND $q$-EULERIAN NUMBERS
}

\author{
WILLIAM DUGAN, SAM GLENNON, PAUL E. GUNNELLS, AND EINAR STEINGRÍMSSON
}

\begin{abstract}
Maxmin trees are labeled trees with the property that each vertex is either a local maximum or a local minimum. Such trees were originally introduced by Postnikov [12, who gave a formula to count them and different combinatorial interpretations for their number. In this paper we generalize this construction and define tiered trees by allowing more than two classes of vertices. Tiered trees arise naturally when counting the absolutely indecomposable representations of certain quivers, and also when one enumerates torus orbits on certain homogeneous varieties. We define a notion of weight for tiered trees and prove bijections between various weight 0 tiered trees and other combinatorial objects; in particular order $n$ weight 0 maxmin trees are naturally in bijection with permutations on $n-1$ letters. We conclude by using our weight function to define a new $q$-analogue of the Eulerian numbers.
\end{abstract}

\section{INTRODUCTION}

1.1. Let $T$ be a tree with vertices labeled by the ordered set $\{1, \ldots, n\}$. We say $T$ is a maxmin tree if for any vertex $v$, the labels of its neighbors are either all less than or all greater than that of $v$. Such trees were introduced by Postnikov [12]; he called them intransitive trees, since they satisfy the property that for any triple $1 \leq i<j<k \leq n$, the pairs $\{i, j\}$ and $\{j, k\}$ cannot both be edges of $T$. These trees first appeared in the study of hypergeometric systems attached to root systems [9], and were later connected to a variety of combinatorial objects:

- regions of the Linial hyperplane arrangement (the affine arrangement in $\mathbb{R}^{n}$ defined by the equations $x_{i}-x_{j}=1,1 \leq i<j \leq n$ );

- local binary search trees (labeled plane binary trees with the property that every left child has a smaller label than its parent and every right child has a larger label than its parent);

- and semiacyclic tournaments (directed graphs on the set $\{1, \ldots, n\}$ such that in every directed cycle, there are more edges $(i, j)$ with $i<j$ than with $i>j$ ).

Date: 8 Feb 2017.

2010 Mathematics Subject Classification. Primary 05C05; Secondary 05A05, 05 C31.

Key words and phrases. Maxmin trees, intransitive trees, Eulerian numbers, $q$-Eulerian numbers, nonambiguous trees, permutations.

This work was partially supported by NSF grant DMS 1501832. We thank the referees for helpful comments. 
For more details, we refer to [6, [7, 12, 13, 16].

1.2. In this paper, we consider a generalization of Postnikov's trees called tiered trees. Instead of two classes of vertices, maxima and minima, we allow more classes. More precisely, a tiered tree with $m \geq 2$ tiers is a tree on the vertex set $V=\{1, \ldots, n\}$ with a map $t$ from $V$ to an ordered set with $m$ elements. We require that if $v$ is a vertex adjacent to $v^{\prime}$ with $v>v^{\prime}$, then $t(v)>t\left(v^{\prime}\right)$.

Tiered trees naturally arise in two a priori unrelated geometric counting problems [11:

- counting absolutely irreducible representations of the supernova quivers (quivers arising in the study of the moduli spaces of certain irregular meromorphic connections on trivial bundles over $\mathbb{P}^{1}[2]$ ), and

- counting certain torus orbits on partial flag varieties of type $A$ over finite fields, namely those orbits with trivial stabilizers.

These contexts also motivate defining a weight function on tiered trees with values in $\mathbb{Z}_{\geq 0}$. We define weights of tiered trees and show that the weights are related to the Tutte polynomials of certain graphs (Theorem 2.9). We show that weight 0 trees of the two extreme types of tiering functions - maxmin trees, which have two tiers, and fully tiered trees, in which every vertex lies on a different tier-are related to other combinatorial objects, namely permutations sorted by their descents and the complete nonambiguous trees of Aval-Boussicault-Bouvel-Silimbani [1]. Finally we use the weights of maxmin trees to define a weight for permutations, which leads to a version of $q$-Eulerian numbers different from those studied by Carlitz, Stanley, and Shareshian-Wachs.

1.3. Here is a guide to the sections of this paper. In $\oint 2$ we define tiered trees and their weights. We also discuss the connection between the weight and geometry in the case of counting torus orbits. In $\$ 3$ we discuss the enumeration of tiered trees. The next two sections give combinatorial interpretations of the two extreme cases of weight zero trees: $\$ 4$ treats weight zero maxmin trees, and \$5 treats weight zero fully tiered trees. Finally in $\S[$ we use weighted maxmin trees to define a notion of weight for permutations and our $q$-Eulerian polynomials. We conclude with a discussion of some open questions.

\section{TiERED TREES AND WEIGHTS}

2.1. For any $n$ let $\llbracket n \rrbracket$ be the finite set $\{1, \ldots, n\}$. Let $G$ be a graph with vertices $V$ labeled by $\llbracket n \rrbracket$ and let $m \geq 2$ be an integer. We say $G$ is tiered with $m$ levels if there is a surjective function $t: V \rightarrow \llbracket m \rrbracket$ such that $t(v) \neq t\left(v^{\prime}\right)$ for any adjacent vertices $v, v^{\prime}$, and such that if $v, v^{\prime}$ are adjacent and $v>v^{\prime}$, then $t(v)>t\left(v^{\prime}\right)$. We call $t$ a tiering function and say $G$ is tiered by $t$. Given $i \in \llbracket m \rrbracket$, we say that the 
vertices $v$ with $t(v)=i$ are at tier $i$. Any tiered graph determines a composition $p=\left(p_{1}, \ldots, p_{m}\right)$ of $|V|$ into $m$ parts by putting $p_{k}=\left|t^{-1}(k)\right|$. We call $p$ the tier type.

2.2. Definition. A tiered tree $T$ with tiering function $t$ is a labeled tree tiered by $t$.

Thus a tiered tree with $m$ levels can be visualized as a tree with its vertices placed at $m$ different heights. Vertices at the same height can never be adjacent, and if two vertices at different heights are adjacent, we require the label of the higher vertex to be larger than that of the lower vertex. Note that this does not mean that the maximal label must occupy the top tier, nor does it mean that the minimal label must occupy the bottom tier. Figure 1 shows a tree tiered with four levels and with tier type $(6,4,2,3)$.

We say a tree is fully tiered if $m=|V|$, and is a maxmin tree if $m=2$. For maxmin trees, it is natural to call the vertices in the top tier maxima and those in the bottom tier minima. Figure 2 shows all the maxmin trees on 4 vertices, grouped according to the number of maxima they have. Similarly, Figure 3 shows the fully tiered trees on 3 vertices.

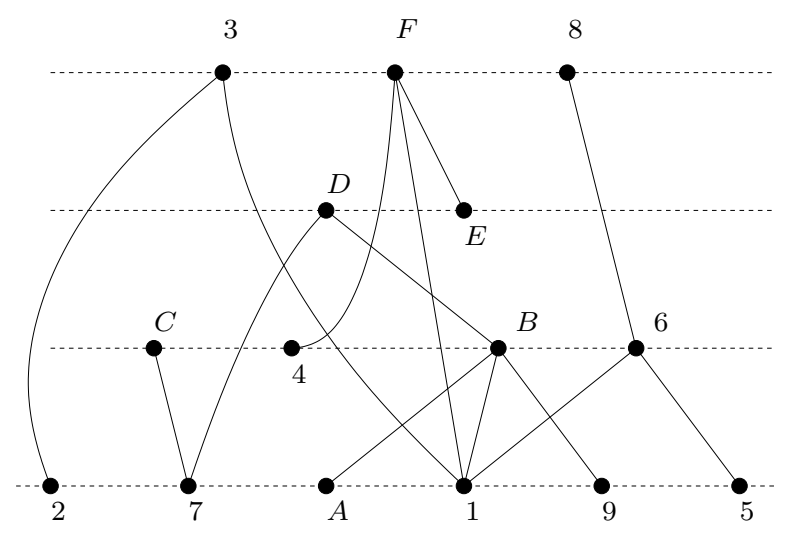

Figure 1. A tiered tree with 4 levels and 15 vertices. The tier type is $(6,4,2,3)$.

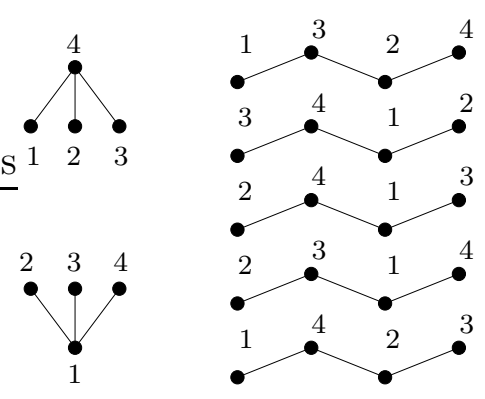

Figure 2. All maxmin trees on 4 vertices. 

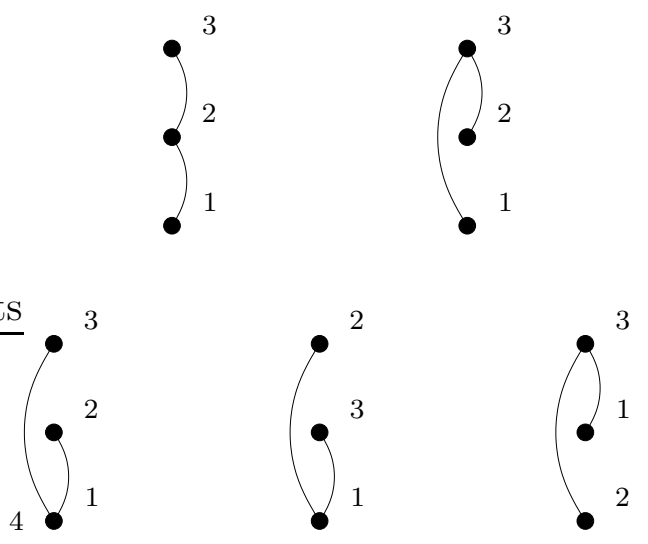

FiguRe 3. All fully tiered trees on 3 vertices.

For any tier type $p$ let $\mathcal{T}_{p}$ be the corresponding set of tiered trees, and let $\mathcal{T}=\cup_{p} \mathcal{T}_{p}$. Our goal now is to define a weight function $w: \mathcal{T} \rightarrow \mathbb{Z}_{\geq 0}$.

2.3. Definition. Let $T \in \mathcal{T}$ have tiering function $t$. The weight $w(T)$ of $T$ is defined as follows:

(i) If $|T|=1$, i.e. if $T$ consists of a single vertex, we put $w(T)=0$.

(ii) If $|T|>1$, let $v$ be the vertex with the smallest label, and let $T_{1}, \ldots, T_{l}$ be the connected components of the forest obtained by deleting $v$ from $T$. We put

$$
w(T)=\sum_{i=1}^{l}\left(w_{i}+w\left(T_{i}\right)\right)
$$

where the integers $w_{i}$ are defined as follows. For each $T_{i}$, let $u_{i} \in T_{i}$ be the vertex that was connected to $v$. We have $t\left(u_{i}\right)>t(v)$ since $v$ had the minimal label. We define $w_{i}$ to be the cardinality of the set

$$
R_{i}=\left\{u_{j} \in T_{i} \mid t\left(u_{j}\right)>t(v) \text { and } u_{j}<u_{i}\right\} .
$$

Thus $w_{i}$ records the (zero-indexed) position of the vertex $u_{i}$ in the ordered list of all those vertices in $T_{i}$ that could have been connected to $v$.

2.4. Example. We give a detailed example of computing the weight of a tiered tree by computing $w(T)$ for the tree $T$ in Figure 1. First we delete 1 and obtain the forest $\left\{T_{i} \mid 1 \leq i \leq 4\right\}$ shown in Figure 4. We have $w(T)=\sum_{i=1}^{4}\left(w_{i}+w\left(T_{i}\right)\right)$, and we consider each component in turn:

(i) We have $w\left(T_{1}\right)=0$, since all tiered trees with only two vertices have weight 0 . Also $w_{1}=0$ since only vertex 3 can be joined to 1 . 
(ii) We have $w\left(T_{2}\right)=1$, since the minimal vertex 4 is connected to $F$ and could have been connected to $E$. We have $w_{2}=2$, since 1 could have been connected to any of $4<E<F$, and is connected to $F$.

(iii) This component is the most complicated. If we delete 7 , we see that the tree $\{C\}$ has weight 0 as does the tree $T^{\prime}=\{9, A, B, D\}$. Furthermore 7 is joined in $T^{\prime}$ to $D$ instead of $B$. Thus altogether we have $w\left(T_{3}\right)=1$. Finally 1 is joined to $B$, the smallest possible vertex it could have been connected to, so $w_{3}=0$.

(iv) Finally $w\left(T_{4}\right)=0$, and 1 is joined to the smallest possible vertex 6 , so $w_{4}=0$. Taking all these contributions together, we find

$$
w(T)=0+0+2+1+1+0+0=4 .
$$

2.5. Example. One can check that all the trees in Figure 2 have weight 0 except for the tree on the bottom right, which has weight 1 . Indeed, after deleting 1 from this tree, we obtain a single tree $T_{1}$ with vertices $\{2,3,4\}$. We have $w\left(T_{1}\right)=0$ and $w_{1}=1$, since 1 was originally connected to 4 and not 3 . It is easy to check that there is a unique maxmin tree on $n$ vertices with 1 maximum and a unique one with $n-1$ maxima, and that the weights of both of these trees are 0 . This accounts for all the trees in Figure 2,

Similarly, the weights of the trees in Figure 3 are all 0 except for the upper right tree, which has weight 1 . In this case, deleting 1 yields the weight 0 tree with vertices $\{2,3\}$, and again $w_{1}=1$ since 1 could have been connected to 2 instead of 3 .

2.6. Example. Table 1 gives a short table of the polynomials

$$
P_{p}(q)=\sum_{T \in \mathcal{T}_{p}} q^{w(T)}
$$

for various tier types $p$. The apparent coincidences, such as $P_{(1,1,1)}=P_{(2,2)}$ and $P_{(1,1,2)}=P_{(2,3)}$ can be explained geometrically via the material in $\$ 2.10$.

2.7. Let $T$ be a tiered tree with respect to $t$. The pair $(T, t)$ determines a unique maximal tiered graph $K_{t}$ containing $T$ as a spanning tree. We simply add all edges to $T$ between vertices $v, v^{\prime}$ with $v>v^{\prime}$ and $t(v)>t\left(v^{\prime}\right)$. We call $K_{t}$ the complete tiered graph $K_{t}$ of order $n$ with respect to $t$. By construction $K_{t}$ is connected. It turns out that the weight of $T$ can be interpreted via the Tutte polynomial of $K_{t}$. We recall the definition of this polynomial in the form we need (cf. [10, Exercise 15.15]).

Let $G$ be a graph with a total ordering of its edge set, let $T \subset G$ be a spanning tree, and let $e$ be an edge of $G$. We say that $e$ is internally active with respect to $T$ if it is contained in $T$ and is the least element in the cut of $G$ determined by $e$ and $T$. We say that $e$ is externally active with respect to $T$ if it is not contained in $T$ and if it is the least element in the unique cycle determined by $T \cup e$. The number 


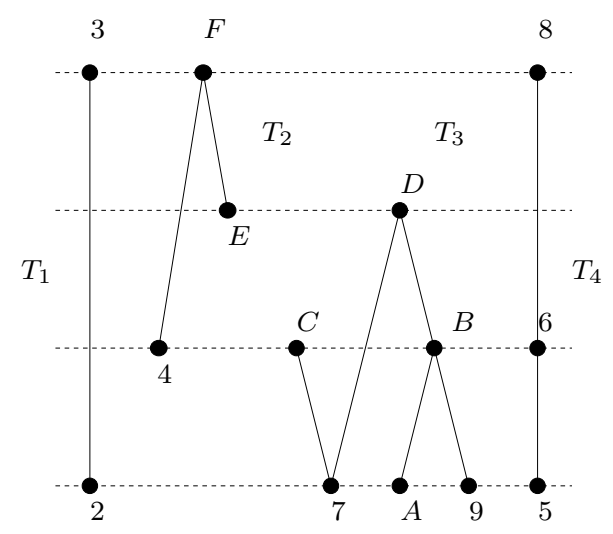

FiguRE 4.

of edges internally (respectively, externally) active with respect to $T$ is called the internal (resp., external) activity of $T$ in $G$.

2.8. Definition. The Tutte polynomial $T_{G}(x, y)$ is defined by

$$
T_{G}(x, y)=\sum_{i, j} t_{i j} x^{i} y^{j}
$$

where $t_{i j}$ is the number of spanning trees of $G$ of internal activity $i$ and external activity $j$.

2.9. Theorem. Let $T$ be a tiered tree, and let $K_{t}$ be the complete tiered graph containing it. Then the weight $w(T)$ is equal to its external activity in $K_{t}$, where the edges of $K_{t}$ are ordered lexicographically by their endpoints.

Proof. We first prove that, in the algorithm described in Definition 2.3, $w_{i}$ is equal to the number of externally active edges in $K_{t}$ that connect $v$ to an element of $T_{i}$.

To see this, let $u$ be a vertex satisfying

$$
u \in\left\{u^{\prime} \in T_{i} \mid t\left(u^{\prime}\right)>t(v) \text { and } u^{\prime} \neq u_{i}\right\},
$$

and consider the edge $e_{v u}$ of $K_{t}$ connecting $v$ to $u$. Because $T$ is a tree and is connected, we have that $e_{v u} \notin T$ (for if it was an edge in $T$ then $T$ would contain a cycle). This unique cycle of $T \cup e_{v u}$ contains $e_{v u}, e_{v u_{i}}$, and no other edges containing $v$. Since $e_{v u}$ and $e_{v u_{i}}$ are the first two edges in the cycle lexicographically, $e_{v u}$ is externally active if and only if $u<u_{i}$, i.e. $u$ lies in the set $R_{i}$ from (1). Hence the cardinality of $R_{i}$ is equal to the number of externally active edges connecting $v$ to $T_{i}$, and our claim is proved.

The full result now follows from our claim, because the sum of the $w_{i}$ 's obtained after deleting the vertex $v$ will now equal the number of externally active edges that have $v$ as the lower of their two vertices. 
2.10. We conclude this section by giving more details about the geometric origin of the weight function. We focus on the connection to counting torus orbits, which is simpler to explain than the connection to representations of quivers. For more details, we refer to [11].

Let $q$ be a prime power and let $\mathbb{F}_{q}$ be the finite field with $q$ elements. Let $G(k, n)$ be the Grassmannian of $k$ planes in $\mathbb{F}_{q}^{n}$. The maximal torus $T \simeq\left(\mathbb{F}_{q}^{\times}\right)^{n-1} \subset \mathrm{PGL}_{n}\left(\mathbb{F}_{q}\right)$ acts on $G(k, n)$ via the standard action of $\mathrm{GL}_{n}\left(\mathbb{F}_{q}\right)$ on $\mathbb{F}_{q}^{n}$. Let us call a $T$-orbit $O \subset G(k, n)$ maximal if its stabilizer is trivial.

Let $O_{k, n}(q)$ be the number of maximal $T$-orbits in $G(k, n)$, as a function of $q$. Then we have (cf. [11, Theorem 3.15])

$$
O_{k, n}(q)=P_{p}(q)=\sum_{T \in \mathcal{T}_{p}} q^{w(T)}
$$

where the sum is taken over tiered trees of type $p=(k, n-k)$.

For instance, the variety $G(1, n)$ is just the projective space $\mathbb{P}^{n-1}$, which is a toric variety. The unique dense $T$-orbit in $G(1, n)$ is maximal and is the only maximal orbit. The equation (2) becomes $O_{1, n}(q)=1$, and indeed there is a unique maxmin tree with $n$ vertices and one maximum (cf. Figure 2 for $n=4$ ).

For a more complicated example, consider the Grassmannian $G(2,4)$. This is not a toric variety and has a more intricate collection of $T$-orbits. See for instance [8, Figure $1]$, which over $\mathbb{C}$ shows the images of the different types of orbits under the moment map; the maximal orbits are those whose moment map image is full-dimensional.

To count the orbits over $\mathbb{F}_{q}$, we can picture 2-planes in a 4-dimensional vector space $V$ via lines in $\mathbb{P}^{3}=\mathbb{P}^{3}(V)$. We claim there are two types of maximal $T$-orbits, depicted in Figure 5). One, as seen on the left, is given by lines in $\mathbb{P}^{3}$ not passing through any of the four $T$-fixed points in $\mathbb{P}^{3}$ (the solid black dots). Any such line $L$ determines four points (the grey dots), which are the intersection points of $L$ with the $T$-fixed planes. The $T$-action preserves the cross-ratio $\lambda$ of these points; since the points are distinct, $\lambda$ is an element of $\mathbb{F}_{q}$ different from 0 or 1 . Thus there are $q-2$ orbits of this type. The other type of orbit corresponds to points passing through a single $T$-fixed line and not meeting a $T$-fixed point. Any such line lies in a unique maximal orbit determined by the fixed line it meets. Thus we have 6 such orbits. The expression (2) becomes $O_{2,4}(q)=q+4$, which agrees with Figure 2 .

We remark that although (2) enumerates maximal $T$-orbits, there is not a bijection between maxmin trees and the orbits. We also remark that general tiered trees can be used to count orbits in partial flag varieties, where the partition type of the flag corresponds to the tier type of the tiering function. Table 1 gives a short list of the corresponding orbit counts.

2.11. Remark. One can show, using geometric results from [11], that the polynomials $P_{p}(q)$ depend only on the partition determined by the tier type $p$, and not the order of its parts. Thus Table 1 gives a complete list of the generating functions for 
weighted tiered trees with up to 6 vertices. We do not know an elementary proof of this fact.
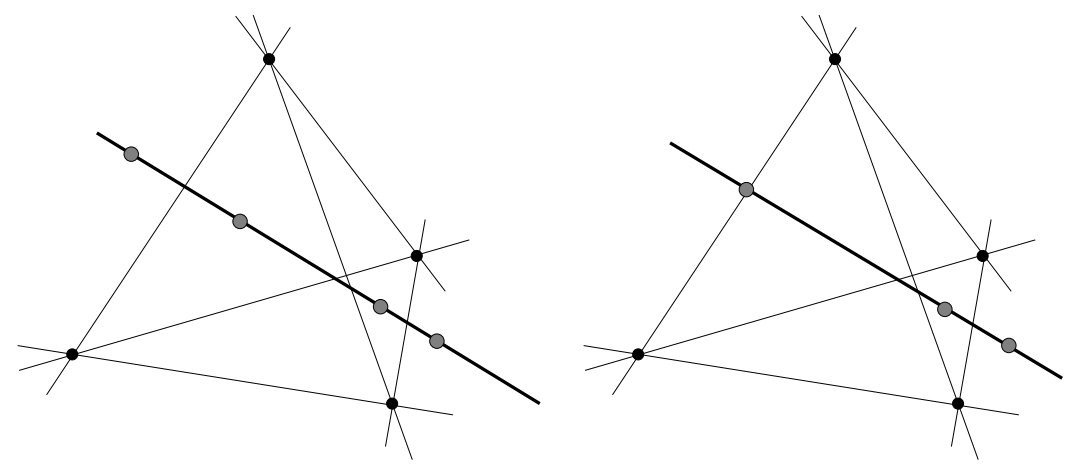

Figure 5. The two types of maximal $T$-orbits in $G(2,4)$. There are $q-2$ of the left type and 6 of the right type, giving $q+4$ altogether.

\begin{tabular}{|l||l|}
\hline Tier type $p$ & $P_{p}(q)=\sum_{T \in \mathcal{T}_{p}} q^{w(T)}$ \\
\hline \hline$(1,1,1)$ & $q+4$ \\
\hline$(2,2)$ & $q+4$ \\
$(1,1,2)$ & $q^{2}+5 q+11$ \\
$(1,1,1,1)$ & $q^{3}+6 q^{2}+20 q+33$ \\
\hline$(2,3)$ & $q^{2}+5 q+11$ \\
$(1,1,3)$ & $q^{3}+6 q^{2}+16 q+26$ \\
$(1,2,2)$ & $q^{4}+6 q^{3}+22 q^{2}+51 q+66$ \\
$(1,1,1,2)$ & $q^{5}+7 q^{4}+28 q^{3}+78 q^{2}+152 q+171$ \\
$(1,1,1,1,1)$ & $q^{6}+8 q^{5}+35 q^{4}+111 q^{3}+260 q^{2}+453 q+456$ \\
\hline$(2,4)$ & $q^{3}+6 q^{2}+16 q+26$ \\
$(3,3)$ & $q^{4}+6 q^{3}+22 q^{2}+51 q+66$ \\
$(1,1,4)$ & $q^{4}+7 q^{3}+22 q^{2}+42 q+57$ \\
$(1,2,3)$ & $q^{6}+7 q^{5}+29 q^{4}+85 q^{3}+190 q^{2}+308 q+302$ \\
$(2,2,2)$ & $q^{7}+7 q^{6}+30 q^{5}+97 q^{4}+243 q^{3}+487 q^{2}+719 q+627$ \\
$(1,1,1,3)$ & $q^{7}+8 q^{6}+36 q^{5}+114 q^{4}+281 q^{3}+549 q^{2}+801 q+718$ \\
$(1,1,2,2)$ & $q^{8}+8 q^{7}+37 q^{6}+127 q^{5}+346 q^{4}+766 q^{3}+1378 q^{2}+1882 q+1533$ \\
$(1,1,1,1,2)$ & $q^{9}+9 q^{8}+45 q^{7}+164 q^{6}+479 q^{5}+1154 q^{4}+2327 q^{3}+3868 q^{2}+$ \\
& $\quad 4957 q+3784$ \\
$(1,1,1,1,1,1)$ & $q^{10}+10 q^{9}+54 q^{8}+209 q^{7}+649 q^{6}+1681 q^{5}+3691 q^{4}+6921 q^{3}+$ \\
& $10805 q^{2}+13139 q+9460$ \\
\hline
\end{tabular}

TABLE 1. Tier types and the corresponding sums over weighted trees. 


\section{Counting tiered trees}

In this section we will show how to enumerate tiered trees; our approach closely follows that of Postnikov [12].

To this end, it will be convenient to extend the notion of tiered trees somewhat. Let $T$ be a tree equipped with a tiering function $t: T \rightarrow \llbracket m \rrbracket$, where $m \geq 2$. If $t$ is surjective, we will say that $T$ is properly tiered. Otherwise, if $t$ is not surjective, we will say that $T$ is improperly tiered if at least two elements of $\llbracket m \rrbracket$ are in the image of $t$. (Thus we never consider tiering functions with one element in their image.) In particular, if $T$ is improperly tiered and has the maximum number of empty tiers, then $T$ canonically has the structure of a maxmin tree.

Let $T_{n, m}$ be the number of properly and improperly tiered trees on $n$ vertices with $m$ tiers. For fixed $m$ let $T_{m}$ be the exponential generating function of the $T_{n, m}$ :

$$
T_{m}(x)=\sum_{n \geq 1} T_{n+1, m} \frac{x^{n}}{n !} .
$$

3.1. Proposition. The generating function $T_{m}(x)$ satisfies the functional equation

$$
T_{m}(x)=\sum_{k=1}^{m} e^{\frac{(m-k) x}{m} \cdot\left(1+T_{m}(x)\right)} .
$$

Proof. It is convenient to consider rooted trees instead. Let us say that a rooted tiered tree is $M_{i}$-rooted if its root lies in the $i$ th tier. Then it follows from Remark 2.11 that given $m$ and $n$, the total number $M_{i, n, m}$ of $M_{i}$-rooted trees is

$$
M_{i, n, m}=\frac{n}{m} \cdot T_{n, m}
$$

Let us put $M_{i, 1, m}=1$, and let $M_{i}(t)$ be the exponential generating function for the $M_{i, n, m}$ :

$$
M_{i}(t):=\sum_{n \geq 1} M_{i, n, m} \frac{t^{n}}{n !}
$$

Then using (3), we have

$$
M_{i}(t)=\frac{t}{m}\left(1+T_{m}(t)\right) .
$$

In particular, the generating function $M_{i}(t)$ is independent of $i$.

Now we consider how to create a tiered tree on $n$ vertices with tiering function taking values in $\llbracket m \rrbracket$. We claim that $T_{m}(x)$ will satisfy the relation

$$
T_{m}(x)=e^{M_{1}(x)+M_{2}(x)+\cdots+M_{m-1}(x)}+e^{M_{1}(x)+\cdots+M_{m-2}(x)}+\cdots+e^{M_{1}(x)} .
$$

To see this, suppose first that $m=2$, as in the case of maxmin trees. Then, as shown in [12, we create a maxmin tree on $n$ vertices by taking all possible forests of rooted trees with $n-1$ vertices and with root a minimum and by attaching the 
$n$th vertex - a maximum - to the root. Note that the new node must be a maximum because it has the largest label. The total number of ways to do this is given by $T_{2}(x)=e^{M_{1}(x)}$.

Now suppose we want to do the same for $m=3$. We start with all possible forests having $n-1$ vertices, but now we have a few cases to consider. Although the $n$th node we use to connect the forests together has the highest label, we can still form a tree in one of two ways. First, if we place vertex $n$ in the highest tier, it can form a valid tiered tree by being connected to trees rooted either in the second tier or those whose roots are in the first. The number of ways to do this is $e^{M_{1}(x)+M_{2}(x)}$. On the other hand, if we place vertex $n$ in the middle tier, then it can still be used to form a valid tiered tree, but only by connecting to trees rooted in the lowest tier. Hence the number of ways for this to happen is given by $e^{M_{1}(x)}$. Combining these cases, we find that for $m=3$ we have

$$
T_{3}(x)=e^{M_{1}(x)+M_{2}(x)}+e^{M_{1}(x)}
$$

If we continue this process by adding more tiers, by similar arguments we arrive at (5). In particular the term

$$
e^{M_{1}(x)+\cdots+M_{k-1}(x)}
$$

in (15) counts the contribution of those rooted tiered trees with root $n$ in the tier $k$.

Finally, using the fact shown above that all $M_{i}(x)$ are equal, say to $M_{1}(x)$, and applying (41) we find

$$
T_{m}(x)=e^{\frac{(m-1) x}{m} \cdot\left(1+T_{m}(x)\right)}+e^{\frac{(m-2) x}{m} \cdot\left(1+T_{m}(x)\right)}+\cdots+e^{\frac{x}{m} \cdot\left(1+T_{m}(x)\right)}
$$

as desired.

3.2. Theorem. The total number $T_{n, m}$ of tiered trees (proper and improper) with $n$ vertices and with $m$ tiers is given by

(6)

$$
T_{n, m}=\frac{1}{n m^{n-1}} \sum_{k_{i} \geq 0}\left(\begin{array}{c}
n \\
k_{1}, k_{2}, \ldots, k_{m}
\end{array}\right)\left((m-1) \cdot k_{1}+(m-2) \cdot k_{2}+\cdots+k_{m-1}\right)^{n-1} \text {. }
$$

We remark that substituting $m=2$ into (6) yields Postnikov's expression for the number of maxmin trees with $n$ vertices:

$$
T_{n, 2}=\frac{1}{n 2^{n-1}} \cdot \sum_{k=1}^{n}\left(\begin{array}{l}
n \\
k
\end{array}\right) k^{n-1}
$$

Proof. By Proposition 3.1, we have

$$
T_{m}(x)=e^{\frac{(m-1) x}{m} \cdot\left(1+T_{m}(x)\right)}+e^{\frac{(m-2) x}{m} \cdot\left(1+T_{m}(x)\right)}+\cdots+e^{\frac{x}{m} \cdot\left(1+T_{m}(x)\right)} .
$$

Let $D(x)=x\left(1+T_{m}(x)\right)$. Then $T_{m}(x)=D(x) / x-1$, and substituting in (17) we find

$$
D(x)=x\left(e^{\frac{m-1}{m} \cdot D(x)}+e^{\frac{m-2}{m} \cdot D(x)}+\cdots+e^{\frac{1}{m} \cdot D(x)}+1\right) .
$$


We can now use Lagrange inversion to find the coefficients of $T_{m}(x)$. Recall that this says that a formal power series $f(x)=a_{1} x+a_{2} x^{2}+\cdots, a_{1} \neq 0$ has compositional inverse $f^{-1}(x)=b_{1} x+b_{2} x^{2}+\cdots$ whose coefficients satisfy

$$
b_{n}=\frac{1}{n}\left[x^{-1}\right]\left(f(x)^{-n}\right),
$$

where for any formal power series $\left[x^{k}\right](f(x))$ denotes the coefficient of $x^{k}$ (cf. [18]). Applying this to our series, we find

$\left[x^{n}\right] D(x)=\frac{1}{n m^{n-1}} \sum\left(\begin{array}{c}n \\ k_{1}, k_{2}, \ldots, k_{m}\end{array}\right) \cdot \frac{\left((m-1) \cdot k_{1}+(m-2) \cdot k_{2}+\cdots+k_{m-1}\right)^{n-1}}{(n-1) !}$.

But the coefficients of $D(x)$ are just those of $T_{m}(x)$ shifted, which implies

$$
T_{n, m}=\frac{1}{n m^{n-1}} \sum\left(\begin{array}{c}
n \\
k_{1}, k_{2}, \ldots, k_{m}
\end{array}\right) \cdot\left((m-1) \cdot k_{1}+(m-2) \cdot k_{2}+\cdots+k_{m-1}\right)^{n-1} .
$$

This completes the proof.

Let $P_{n, m}$ denote the properly tiered trees on $n$ vertices with $m$ tiers. We can easily evaluate $P_{n, m}$ using the principle of inclusion-exclusion:

3.3. Proposition. We have

$$
P_{n, m}=\sum_{k=0}^{m-2}(-1)^{k}\left(\begin{array}{c}
m \\
m-k
\end{array}\right) T_{n, k} .
$$

Proof. The proof is by induction on the number of tiers $m$. We have $P_{n, 2}=T_{n, 2}$ by definition. For $m=3$, the only possible improperly tiered trees are those that take up two tiers, leaving the third empty. Thus we have $P_{n, 3}=T_{n, 3}-\left(\begin{array}{l}3 \\ 2\end{array}\right) P_{n, 2}$, and since $P_{n, 2}=T_{n, 2}$, we find $T_{n, 3}-\left(\begin{array}{l}3 \\ 2\end{array}\right) T_{n, 2}$.

In general, we have

$$
P_{n, m}=T_{n, m}+\sum_{k=1}^{m-2}(-1)^{k}\left(\begin{array}{c}
m \\
m-k
\end{array}\right) P_{n, m-k}
$$

To pass to (8) , one substitutes the previously obtained expressions for $P_{n, k}, k<m$ and uses elementary properties of binomial coefficients.

We conclude this section by giving a short table (Table 21) of the numbers $T_{n, m}$ and $P_{n, m}$. We remark that the $P_{n, m}$ can also be recovered from the polynomials in Table 1 by substituting $q=1$ and combining various terms. For instance $P_{4,4}=P_{(1,1,1,1)}(1)=$ 60 , and $P_{4,3}=3 P_{(1,1,2)}(1)=3 \cdot 17=51$. 


\begin{tabular}{|c|c|c|c||c|c|c|c||c|c|c|c||}
\hline$n$ & $m$ & $T_{n, m}$ & $P_{n, m}$ & $n$ & $m$ & $T_{n, m}$ & $P_{n, m}$ & $n$ & $m$ & $T_{n, m}$ & $P_{n, m}$ \\
\hline \hline 3 & 1 & 0 & 0 & 4 & 4 & 306 & 60 & 6 & 1 & 0 & 0 \\
3 & 2 & 2 & 2 & 5 & 1 & 0 & 0 & 6 & 2 & 246 & 246 \\
3 & 3 & 11 & 5 & 5 & 2 & 36 & 36 & 6 & 3 & 8868 & 8130 \\
4 & 1 & 0 & 0 & 5 & 3 & 693 & 585 & 6 & 4 & 80496 & 46500 \\
4 & 2 & 7 & 7 & 5 & 4 & 4304 & 1748 & 6 & 5 & 400200 & 83940 \\
4 & 3 & 72 & 51 & 5 & 5 & 16274 & 1324 & 6 & 6 & 1414050 & 46620 \\
\hline
\end{tabular}

TABle 2. The numbers $T_{n, m}$ and $P_{n, m}$.

\section{Weight Zero maXmin tREes And EulERIAN Numbers}

Define a polynomial $\mathscr{T}_{n}(x, q)$ by

$$
\mathscr{T}_{n}(x, q)=\sum_{T} x^{k(T)} q^{w(T)}
$$

where $T$ ranges over all maxmin trees with $n$ vertices, and for any tree $T, w(T)$ is its weight and $k(T)$ is its number of maxima. Using Table 1, the first few polynomials are

$$
\begin{aligned}
& \mathscr{T}_{4}=\left(x+x^{3}\right)+x^{2}(q+4), \\
& \mathscr{T}_{5}=\left(x+x^{4}\right)+\left(x^{2}+x^{3}\right)\left(q^{2}+5 q+11\right), \\
& \mathscr{T}_{6}=\left(x+x^{5}\right)+\left(x^{2}+x^{4}\right)\left(q^{3}+6 q^{2}+16 q+26\right)+x^{3}\left(q^{4}+6 q^{3}+22 q^{2}+51 q+66\right) .
\end{aligned}
$$

If we set $q=0$, we find

$$
\mathscr{T}_{n}(x, 0)=\sum_{k=1}^{n-1} A(k-1, n-1) x^{k},
$$

where $A(k, n)$ is the Eulerian number (the number of permutations in $S_{n}$ with $k$ descents). Thus $\mathscr{T}_{n}(x, 0)$ is essentially the Eulerian polynomial.

In fact, it is not difficult to prove the relationship (9) using generating functions. More precisely, the generating function of the Eulerian numbers is well known. Postnikov [12] enumerated all maxmin trees on $n$ vertices, which means he computed the specialization $\mathscr{T}_{n}(x, 1)$. One can modify his result to include the weight parameter $q$ so that setting $q=0$ finds the generating function for the Eulerian numbers.

Our goal in this section is to give a bijective proof of (91). This has the advantage of revealing a connection between permutations and maxmin trees that was previously unknown. It will also enable us later (\$6] $)$ to define a $q$-analogue of the Eulerian numbers.

4.1. Theorem. There is a bijection between permutations in $S_{n}$ with $k$ descents and weight 0 maxmin trees on $(n+1)$ vertices with $(k+1)$ maxima. 
Proof. We define the bijection recursively. We begin by taking the identity permutation in $S_{n}$ to the unique maxmin tree on $(n+1)$ vertices with 1 maximum. Similarly we take the longest element of $S_{n}$, the decreasing permutation, to the unique maxmin tree on $(n+1)$ vertices with $n$ maxima.

For the rest of $S_{n}$, we regard $S_{n}$ as embedded in $S_{n+1}$ as follows. We take any $\pi \in S_{n}$ and represent it as an ordered sequence of $1, \ldots, n$. We then adjoin an $n+1$ on the right and obtain an element of $S_{n+1}$. In other words, we identify $S_{n}$ with the parabolic subgroup of $S_{n+1}$ generated by all simple transpositions other than $(n, n+1)$. We will in fact construct a bijection between permutations in this subgroup of $S_{n+1}$ and maxmin trees on $(n+1)$ vertices.

Thus given $\pi \in S_{n}$ represented as above as an ordered sequence on $1, \ldots, n+1$ with $n+1$ on the right, we break $\pi$ up into the two subsequences appearing to the left and right of 1: we write

$$
\pi=\pi_{L} \cdot 1 \cdot \pi_{R}
$$

where the operator - denotes concatenation. Note that $\pi_{L}$ may be empty, but $\pi_{R}$ contains at least the symbol $n+1$. We further break $\pi_{L}$ into a collection of subsequences

$$
\pi_{L}=\pi_{1} \cdot \pi_{2} \cdots \pi_{l}
$$

as follows. Let $m_{1}$ be the maximum symbol appearing in $\pi_{L}$. Then $\pi_{1}$ consists of the subsequence of $\pi_{L}$ from the beginning to $m_{1}$. We then let $m_{2}$ be the maximum of what's left after deleting $\pi_{1}$, and let $\pi_{2}$ be the corresponding subsequence up to $m_{2}$. We continue this process until $\pi_{L}$ is exhausted.

The result is a collection of ordered sequences $\pi_{1}, \ldots, \pi_{l}$ and $\pi_{R}$ with the following property: the global maximum of each appears in the rightmost position. By induction on the number of vertices, we know how to build a weight 0 maxmin tree with the correct number of maxima and vertices for each of these subsequences (after relabeling them to preserve their permutation patterns). We take the resulting forest of weight 0 maxmin trees and join them together into a maxmin tree for $\pi$ by joining 1 to the smallest maximum available in each connected component. The resulting tree clearly has weight 0 . It is easy to verify that this construction is a bijection with the stated property.

4.2. Example. Consider the element $\pi \in S_{10}$ given by the sequence 8594673201 (we use characters $0, \ldots, 9$ for $\llbracket 10 \rrbracket$ for this example). Note that this permutation has five descents, which occur at 8, 9, 7, 3, and 2. We are thus building a maxmin tree with six maxima. We identify $\pi$ with the permutation in $S_{11}$ obtained by adjoining the character A (hexadecimal 10) on the right: $\pi=8594673201 \mathrm{~A}$. We have

$$
\pi_{L}=85946732, \quad \pi_{R}=1 \mathrm{~A} .
$$

The left permutation becomes broken into four pieces

$$
\pi_{L}=859 \cdot 467 \cdot 3 \cdot 2
$$


which means that we must connect vertex 0 to five maxmin trees. The resulting maxmin tree, which has six maxima, is shown in Figure 6.

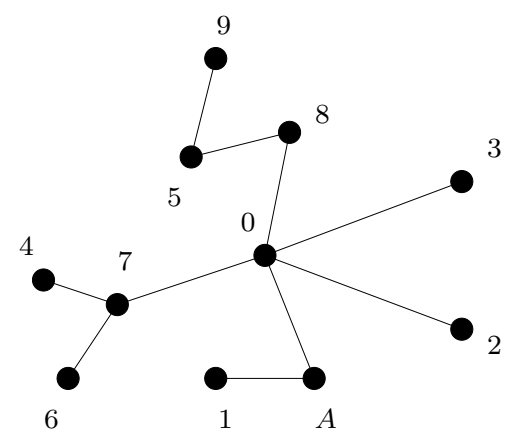

FigURE 6 .

4.3. We conclude this section by connecting the decomposition into subsequences in Theorem 4.1 with the Stirling numbers of the first kind.

4.4. Theorem. The number of permutations in $S_{n}$ that break into $k$ blocks (subsequences) in the decomposition in Theorem 4.1, disregarding the singleton block with the minimum element, equals $n \cdot c(n-1, k)$ where $c(n-1, k)$ is the signless Stirling number of the first kind.

Proof. The signless Stirling number of the first kind, $c(n-1, k)$, counts permutations in $S_{n-1}$ with $k$ cycles when they are written in disjoint cycle notation. Given such a permutation we construct $n$ different permutations in $S_{n}$, each of which breaks into $k$ blocks as in the proof of Theorem 4.1. Namely, we can insert 0 after any one of the $n-1$ letters in the $k$ cycles, or else as a cycle of its own. Once we have done that we write the cycle containing 0 with the 0 first and make that the last cycle in our ordering of the cycles. The remaining cycles we write with their maximum letter last, and order them in decreasing order of their maxima (followed by the cycle containing 0). Erasing the parentheses from the word thus constructed we obtain a permutation in $S_{n}$ whose blocks are precisely the cycles we started with. It is easy to see that this constructs $n$ distinct permutations, and that any permutation in $S_{n}$ is constructed this way.

4.5. Example. If in the permutation with cycle decomposition $(237)(418)(69)(5)$ we insert 0 after the 2 we get the following cycles, ordered as in the proof of Theorem 4.4; (69)(418)(5)(0372). Erasing the parentheses gives the permutation 6941850723, which decomposes into the blocks $69 \cdot 418 \cdot 5 \cdot 372$. Inserting 0 as its own block, on the other hand, gives $(69)(418)(237)(5)(0)$, which yields $69 \cdot 418 \cdot 237 \cdot 5$. 


\section{Weight ZERO FUlLy TIERED TREeS AND COMPLETE NONAMBIGUOUS TREES}

5.1. A tiered tree is called fully tiered if all its vertices sit on distinct levels, i.e. it has tier type $(1, \ldots, 1)$. In this section we describe a bijection between weight 0 fully tiered trees and the complete nonambiguous trees defined by Aval-BoussicaultBouvel-Silimbani [1]. We begin by defining the latter objects, following [1].

5.2. Let $G=\mathbb{Z}_{>0} \times \mathbb{Z}_{>0}$ be the grid of nonnegative lattice points. Given any $v \in G$, let $(X(v), Y(v))$ be its coordinates. For later convenience, in figures we represent points in $G$ by putting $(1,1)$ in the upper-left corner, having the $X$ coordinate increase from left to right, and having the $Y$ coordinate increase from top to bottom. A nonambiguous tree is a subset $A \subset G$ satisfying the following properties:

(i) $(1,1) \in A$. This is the root of the tree.

(ii) Let $p \in A$ be different from the root. Then there exists a unique point $q \in A$ with either (i) $X(q)<X(p)$ and $Y(q)=Y(p)$ or (ii) $X(q)=X(p)$ and $Y(q)<Y(p)$. These possibilities are exclusive: the point $q$ must satisfy one or the other.

(iii) there is no empty line between two given points: if there exists a point $p \in A$ such that $X(p)=x($ resp. $Y(p)=y)$, then for every $x^{\prime}<x\left(\right.$ resp. $\left.y^{\prime}<y\right)$ there exists $q \in A$ such that $X(q)=x^{\prime}\left(\right.$ resp. $\left.Y(q)=y^{\prime}\right)$.

Any subset $A$ satisfying these properties determines a unique binary tree $T(A)$ embedded in $G$ with edges running along the grid lines. More precisely, if $p \in A$ is a point different from the root, then the parent of $p$ is the unique point $q \in A$ preceding $p$ in the same row or column; by condition (iil) above, $q$ is uniquely determined. This motivates the name "nonambiguous:" the tree structure on $A$ can be recovered from the vertex coordinates even if the edges are missing. Figure 7 shows four different nonambiguous trees with 5 vertices.

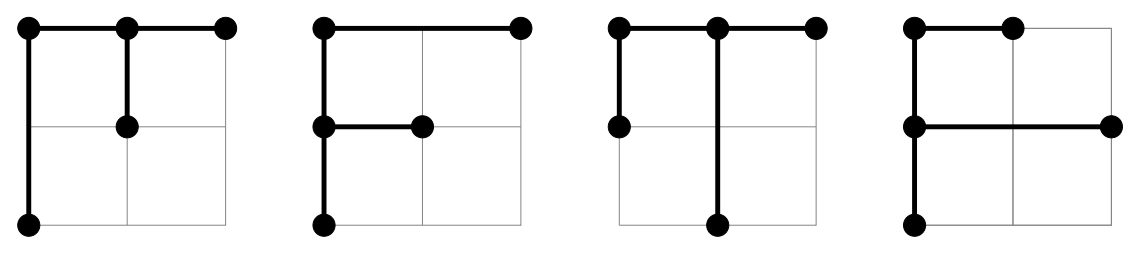

Figure 7. Four nonambiguous trees

5.3. The trees in Figure 7 have the further property of being complete. A complete nonambiguous tree is one that is complete in the usual sense, that is, every point has 0 or 2 children. Any such tree has $2 k+1$ vertices, $k+1$ of which are leaves and $k$ of which are internal. 
Let $b_{k}, k \geq 0$ be the number of complete nonambiguous trees with $k$ internal vertices. The sequence $b_{k}$, which begins

$$
1,1,4,33,456,9460,274800,10643745,530052880,32995478376, \ldots,
$$

appears in OEIS [15] as sequence A002190, and is related to the logarithm of the $J$-Bessel function:

$$
\sum_{k \geq 1} b_{k-1} \frac{x^{k}}{k !^{2}}=-\log J_{0}(2 \sqrt{x}) .
$$

According to [1], complete nonambiguous trees provided the first combinatorial interpretation of the sequence A002190, which was originally defined through (11). The following theorem provides another combinatorial interpretation of this sequence:

5.4. Theorem. There is a bijection between weight 0 fully-tiered trees on $n$ vertices and complete nonambiguous trees with $n-1$ internal vertices.

Proof. We construct the bijection by induction. The unique complete nonambiguous trees with 0 and 1 internal vertices correspond respectively to the unique fully tiered trees with 1 and 2 vertices.

A complete nonambiguous tree $T$ with $n$ internal vertices sits inside the $(n+1) \times$ $(n+1)$ subsquare of $\mathbb{Z}_{>0} \times \mathbb{Z}_{>0}$. Each row and each column contains a single leaf of $T$. The tree $T$ determines a labeling of the $n+1$ levels of the associated fully tiered tree $T^{\prime}$ as follows: if a leaf appears at row $i$ (numbering from the bottom of the figure to the top) and column $j$, then at level $i$ in $T^{\prime}$ we place the vertex $j$ (see Figure 8).

Now consider erasing the first column of $T$, along with all the edges connecting vertices in this column to nodes in greater columns. The result is a forest of complete nonambiguous trees, although each is embedded with roots at different points of $\mathbb{Z}_{>0} \times \mathbb{Z}_{>0}$, and such that their vertex labels are various subsets of $\llbracket n \rrbracket$. By flattening their vertex labels in the obvious way and shifting the roots, each of these trees corresponds to a complete nonambiguous tree. Thus by induction we know the fully tiered trees to which they correspond. This allows us to add the edges they determine to $T^{\prime}$ (using the original vertex labels, not their flattened versions) to obtain a fully tiered forest. To complete $T^{\prime}$ to a weight 0 fully tiered tree, we need to connect the vertex 1 to each connected component, and in doing so we choose the minimal vertex we can for each component. The resulting fully tiered tree clearly has weight 0 (see Figure 9 for a complete example).

We claim this is a bijection. To go backwards, we reverse the process. We take a fully tiered tree $T^{\prime}$ and delete the vertex 1. This gives a forest of fully tiered trees with fewer vertices. We can build complete nonambiguous trees from them and can embed them into $\mathbb{Z}_{>0} \times \mathbb{Z}_{>0}$ using the disjoint sets of rows and columns determined by their labels and by the levels on which the labels appear. The final step is to add the root and the necessary edges from the first column heading right to the roots 
of the smaller trees. Note that these smaller complete nonambiguous trees can be interlaced to form a complete nonambiguous tree exactly because their labels and levels in $T^{\prime}$ form disjoint subsets.

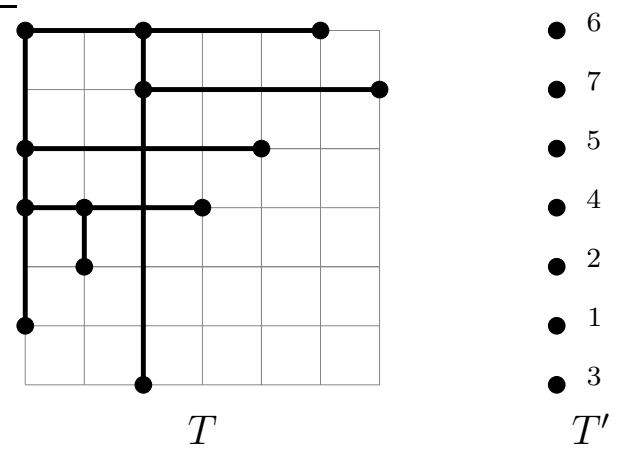

FiguRE 8. Extracting levels for $T^{\prime}$ from a complete nonambiguous tree $T$. For example, the bottommost leaf in $T$ in column 3 puts the label 3 at the bottom tier of $T^{\prime}$.

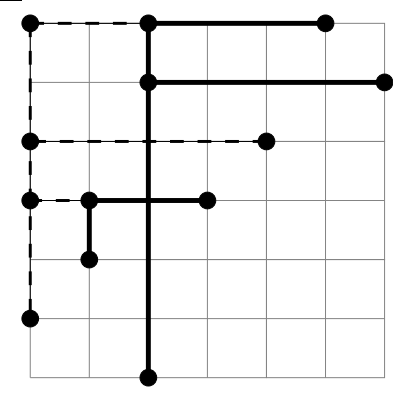

$T$

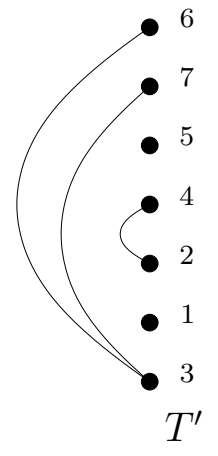

Figure 9. Building a fully tiered tree from a complete nonambiguous tree. On the left, we add edges for the forest obtained by deleting the first column. On the right, we add edges from 1 to the connected components of the forest.

\section{Weights of PERMUtATions AND q-EULERIAN NUMBERS}

Theorem 4.1 shows that order $n$ weight 0 maxmin trees with $k$ maxima are in bijection with elements of $S_{n-1}$ with $k-1$ descents. What can one say about trees of higher weight? In fact, the proof of Theorem 4.1 shows that any maxmin tree of any weight is built from an underlying permutation. Indeed, in the bijection between trees and permutations, one builds a weight 0 tree from a permutation by extracting subpermutations, building weight 0 trees from them, and then by connecting all the 
smaller trees to the minimal vertex canonically: one joins the minimal vertex to the smaller trees by picking maxima with the smallest labels.

Thus it is clear how to modify this construction to produce a tree of higher weight. At any stage, one can connect the current minimal vertex to a larger maximum instead of choosing the smallest maximum available. For instance, in Example 4.2, one could connect 0 to 9 instead of 8 to make a tree of weight 1 . Moreover, this is the tree of largest weight one can make from this permutation. This leads to the following definition:

6.1. Definition. Let $\pi \in S_{n}$. Then the weight $w(\pi)$ of $\pi$ is the largest possible weight of maxmin tree that can be constructed from $\pi$. More precisely, the function $w: S_{n} \rightarrow \mathbb{Z}_{\geq 0}$ is defined by the following recursion:

(i) If $\pi$ is the identity or the longest element in $S_{n}$, then we define $w(\pi)=0$.

(ii) Otherwise, regard $\pi$ as an element of $S_{n+1}$ with the symbol $n+1$ appearing on the right, as in the proof of Theorem 4.1, and let $\pi_{1}, \ldots, \pi_{l}, \pi_{R}$ be the subpermutations extracted there. Then we define

$$
w(\pi)=w\left(\pi_{R}\right)+d_{R}+\sum_{i=1}^{l}\left(w\left(\pi_{i}\right)+d_{i}\right),
$$

where $d_{i}$ (respectively, $d_{R}$ ) is the number of descents in $\pi_{i}$ (respectively, $\pi_{R}$ ).

6.2. Example. Let us consider the permutation $\pi=15 A 86290374 \in S_{11}$. We can complete $\pi$ to 15A86290374B by putting a new maximal element B on the right. Extracting subsequences around the minimal element 0, we find

$$
15 \mathrm{~A} \cdot 8629 \cdot 0 \cdot 374 \mathrm{~B}
$$

Computing $w(\pi)$ proceeds as follows:

- 15A flattens to 012 , which is the identity. We have $w(15 \mathrm{~A})=0$ and $d(15 \mathrm{~A})=$ 0.

- 8629 flattens to 2103, which is the completion of the longest word. We have $w(8629)=0$ and $d(8629)=2$.

- 374B flattens to 0213. A short computation shows $w(374 \mathrm{~B})=1$ (compare with Figure 2 above; the tree on the bottom right corresponds to this permutation). We have $d(374 \mathrm{~B})=1$.

Thus $w(15 \mathrm{~A} 86290374)=(0+0)+(0+2)+(1+1)=4$.

6.3. With our definition of weight, we can define a $q$-analogue of the Eulerian polynomials. Put

$$
E_{n}(x, q)=\sum_{\pi \in S_{n}} x^{d(\pi)} q^{w(\pi)}
$$


Some examples of $E_{n}(x, q)$ are given below.

$$
\begin{aligned}
E_{4}(x, q)= & +x\left(q^{2}+3 q+7\right)+x^{2}\left(q^{2}+4 q+6\right)+x^{3}, \\
E_{5}(x, q)=1 & +x\left(q^{3}+3 q^{2}+7 q+15\right)+x^{2}\left(q^{4}+4 q^{3}+11 q^{2}+25 q+25\right) \\
& +x^{3}\left(q^{3}+5 q^{2}+10 q+10\right)+x^{4} \\
E_{6}(x, q)= & +x\left(q^{4}+3 q^{3}+7 q^{2}+15 q+31\right) \\
& +x^{2}\left(q^{6}+4 q^{5}+11 q^{4}+31 q^{3}+58 q^{2}+107 q+90\right) \\
& +x^{3}\left(q^{6}+5 q^{5}+16 q^{4}+34 q^{3}+76 q^{2}+105 q+65\right) \\
& +x^{4}\left(q^{4}+6 q^{3}+15 q^{2}+20 q+15\right)+x^{5} .
\end{aligned}
$$

6.4. We pause to compare the polynomials $E_{n}(x, q)$ with other $q$-Eulerian polynomials in the literature. Let $[x]_{q}=\left(q^{x}-1\right) /(q-1)$ and for $m \geq 1$ let

$$
\left(\begin{array}{c}
x \\
m
\end{array}\right)_{q}=\frac{\left(q^{x}-1\right)\left(q^{x-1}-1\right) \cdots\left(q^{x-m+1}-1\right)}{(q-1)\left(q^{2}-1\right) \cdots\left(q^{m-1}-1\right)}
$$

Carlitz [4] defined $q$-polynomials $A_{n, s}^{*}(q) \in \mathbb{Z}[q]$ as follows. First we define $A_{n, s}(q)$ by the expansion

$$
[x]_{q}^{n}=\sum_{s=1}^{n} A_{n, s}(q)\left(\begin{array}{c}
x+s-1 \\
n
\end{array}\right)_{q},
$$

and then set $A_{n, s}^{*}(q)=q^{-(n-s)(n-s-1) / 2} A_{n, s}(q)$. The result is still an integral polynomial in $q$. Then Carlitz's $q$-Eulerian polynomial $E_{n}^{\mathrm{C}}(x, q)$ is defined by

$$
E_{n}^{\mathrm{C}}(x, q)=\sum_{d=0}^{n-1} A_{n, d+1}^{*}(q) x^{d}
$$

For example,

$$
\begin{gathered}
E_{3}^{\mathrm{C}}(x, q)=x^{2}+(2 q+2) x+1 \\
E_{4}^{\mathrm{C}}(x, q)=x^{3}+\left(3 q^{2}+5 q+3\right) x^{2}+\left(3 q^{2}+5 q+3\right) x+1 .
\end{gathered}
$$

Stanley [17] defined a different $q$-Eulerian polynomial as follows. For any permutation $\pi$, let $i(\pi)$ be the number of inversions: if $\pi$ is written as an ordered list $\left(a_{1}, \ldots, a_{n}\right)$, then $i(\pi)$ counts the number of pairs $(i, j)$ with $i<j$ and $a_{i}>a_{j}$. Then Stanley defined

$$
E_{n}^{\mathrm{S}}(x, q)=\sum_{\pi \in S_{n}} x^{d(\pi)} q^{i(\pi)}
$$

For example,

$$
\begin{gathered}
E_{3}^{\mathrm{S}}(x, q)=x^{2} q^{3}+x\left(2 q^{2}+2 q\right)+1 \\
E_{3}^{\mathrm{S}}(x, q)=x^{3} q^{6}+x^{2}\left(3 q^{5}+4 q^{4}+3 q^{3}+q^{2}\right)+x\left(q^{4}+3 q^{3}+4 q^{2}+3 q\right)+1 .
\end{gathered}
$$


Finally, Shareshian-Wachs [14] generalized Stanley's definition by considering four different sums of the form

$$
E_{n}^{\mathrm{SW}}(x, q)=\sum_{\pi \in S_{n}} x^{a(\pi)} q^{b(\pi)}
$$

where $a$ and $b$ are various statistics of permutations; one example yields Stanley's polynomial.

6.5. From these examples it is clear that our $E_{n}(x, q)$ is quite different. Indeed, there are many interesting problems to investigate about the combinatorial meaning of these polynomials. We indicate some below, and for the present purposes prove the following results:

6.6. Theorem. There is a bijection between weight 0 permutations on $n$ letters with $k-1$ descents and partitions of $\llbracket n \rrbracket$ into $k$ subsets. Thus such permutations are counted by $\left\{\begin{array}{l}n \\ k\end{array}\right\}$, the Stirling numbers of the second kind.

Proof. We describe an algorithm that constructs a weight 0 permutation given any partition of the set $\llbracket n \rrbracket$ (cf. Example 6.7), and will prove that this provides our bijection.

Let $P$ be a partition of the set $\llbracket n \rrbracket$ into $k$ subsets. Sort each subset into increasing order, and denote the resulting ordered subsets by $\pi_{1}, \pi_{2}, \ldots, \pi_{k}$. We assemble these subpermutations $\pi_{i}$ into a permutation $\pi \in S_{n}$ as follows. Let $\pi_{l}$ be the sublist containing the minimum element 1 , and place it in the rightmost position in $\pi$. Next, sort the remaining $\pi_{i}$ by their respective maxima, and arrange them in order such that their maxima decrease. Finally, adjoin this string of subpermutations to the left of $\pi_{l}$ to complete $\pi$. Because we sorted each $\pi_{i}$ and ordered them with their maxima decreasing, the final permutation $\pi$ has $k-1$ descents.

We also claim that $\pi$ has weight 0 . To see this, first observe that each $\pi_{i}$ in $\pi$ has no descents, so each one has weight 0 . Moreover, each tree constructed from the $\pi_{i}$ contains only a single maximum, so there is no choice as to how we combine them together to construct the tree corresponding to $\pi$ : the maximum in each subtree must be connected to the global minimal element. Hence the maxmin tree corresponding to $\pi$ has weight 0 , and this is the maximum possible weight it could have had.

Finally, notice that the correspondence described is a bijection between partitions of $\llbracket n \rrbracket$ and weight 0 permutations: the steps outlined above demonstrate that each partition determines a unique permutation. Conversely, given the permutation we can recover the original partition of $\llbracket n \rrbracket$ by splitting the permutation at its descent points. This completes the proof.

6.7. Example. Consider the partition of $\llbracket 10 \rrbracket$ into 4 parts given by 25, 6130, 798, 4. To build $\pi$, we sort the parts to obtain $25,0136,789,4$, and then place the 0136 block on the right of $\pi$. The remaining blocks are placed on the left with their 
maxima decreasing: $789,25,4$. The final permutation is

$$
\pi=7892540136
$$

which has 3 descents. The corresponding maxmin tree is shown in Figure 10.

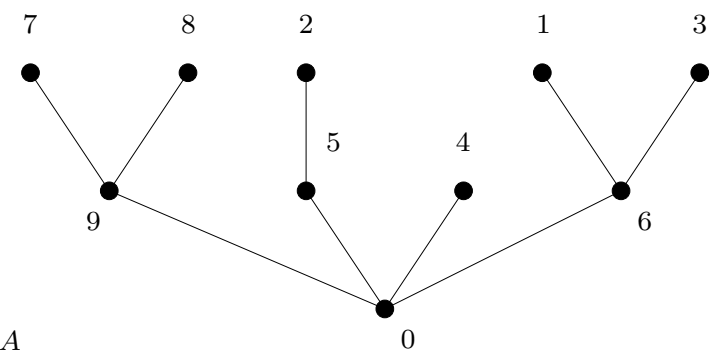

Figure 10. The weight 0 maxmin tree for $\pi=7892540136$

6.8. Corollary. The coefficient of $x$ in $E_{n}(x, q)$ is $q^{n-2}+3 q^{n-3}+7 q^{n-4}+\cdots+\left(2^{n-1}-\right.$ 1 ), and the coefficient of $x^{n-2}$ is $q^{n-2}+\left(\begin{array}{c}n \\ 1\end{array}\right) q^{n-3}+\left(\begin{array}{c}n \\ 2\end{array}\right) q^{n-4}+\cdots+\left(\begin{array}{c}n \\ n-2\end{array}\right)$.

Proof. We prove the first statement; the second can be proved similarly. Let $a(q)=$ $a_{0}+a_{1} q+a_{2} q^{2}+\cdots$ be the coefficient of $x$ in $E_{n}(x, q)$, and let $b(q)=q^{n-2}+3 q^{n-4}+$ $7 q^{n-5}+\cdots+\left(2^{n-1}-1\right)$.

First we observe that if $\pi \in S_{n}$ is a weight $w$ permutation with 1 descent, then it determines a weight $w+1$ permutation $\pi^{\prime} \in S_{n+1}$ with the same number of descents through a "promotion" operation: if $\pi$ is given by the ordered list $\left(a_{1}, \ldots, a_{n}\right)$, then $\pi^{\prime}$ is defined by the list $\left(1, a_{1}+1, \ldots, a_{n}+1\right)$.

We now use this operation to determine the coefficients as follows. By Theorem 6.6, we have $a_{0}=\left\{\begin{array}{c}n \\ 2\end{array}\right\}=2^{n-1}-1=b_{0}$. Applying promotion once, we get $a_{1} \geq\left\{\begin{array}{c}n-1 \\ 2\end{array}\right\}=b_{1}$. Applying promotion a second time, we get $a_{2}=\left\{\begin{array}{c}n-2 \\ 2\end{array}\right\}=b_{2}$, and so on. Hence the difference $c(q)=a(q)-b(q)$ is a nonnegative polynomial in $q$. But one knows that the Euler number $A(1, n)$ equals $2^{n}-n-1$. This implies $c(1)=0$, which means $a(q)=b(q)$. Thus the coefficient of $x$ is $b(q)$ as claimed.

6.9. Theorem 6.6 addresses the constant terms of the $E_{n}(x, q)$. The next result shows that these polynomials are monic and computes their degrees.

6.10. Theorem. The maximum weight of a permutation of length $n$ with $d$ descents is $d(n-1-d)$. This maximum is attained only by the permutation

$$
12 \ldots(n-d-1) n(n-1) \ldots(n-d) .
$$

We will need the following lemma, whose simple proof we leave to the reader:

6.11. Lemma. If a permutation $\pi$ ends in an ascent, and $\pi^{\prime}$ is obtained by removing the last letter from $\pi$, then $\pi^{\prime}$ has the same weight as $\pi$. 
Proof of Theorem 6.10. It is clear that the permutation mentioned in the theorem has the weight claimed. This permutation has an empty $\pi_{L}$. It is straightforward to check that the claim is true for any $d$ and all $n<4$. Using that for an inductive argument, we will prove the theorem for any $n$ and $d$. We first show that if $\pi_{L}$ has two or more components then $\pi$ has smaller weight than a permutation obtained by merging the last two components of $\pi_{L}$ in a certain way. Then we show that a permutation with a single-component $\pi_{L}$ has smaller weight than the permutation obtained by merging that $\pi_{L}$ and $\pi_{R}$ to produce a permutation of the form described in the statement.

Suppose the last two components, $\pi_{C}$ and $\pi_{D}$, of $\pi_{L}$ have lengths $(k+1)$ and $(\ell+1)$, respectively, and that their respective numbers of descents are $a$ and $b$. Because of how we partition, a component cannot end in a descent (since there would have been a split between the letters of that last descent). By Lemma 6.11, the components have the same weight after we remove their respective last letters.

Thus, by the inductive hypothesis, the sum of the maximum possible weights of these two components is

$$
\mathrm{wt}_{2}=a(k-a)+b(\ell-b) .
$$

We will compare this to the maximum weight of a permutation obtained by using all the letters of both components to construct a single component, which must then end in a non-descent, and have $a+b+1$ descents, since there is a descent between the original two components. We construct this component to have its largest letter last (since otherwise it couldn't be a single component), preceded by the largest $a+b+2$ letters left, in decreasing order, so as to get $a+b+1$ descents, and with the remaining letters prepended to this in increasing order. An example is 12376548.

Note that the component thus constructed will still force a break preceding it, in the partition, since its smallest letter is clearly smaller than the last letter of the component preceding the components $\pi_{C}$ and $\pi_{D}$.

By Lemma 6.11, the weight of this newly constructed component is equal to the weight of the permutation of length $k+\ell+1$ obtained by removing its last letter. That weight is

$$
\mathrm{wt}_{1}=(a+b+1)(k+\ell-a-b-1)
$$

A straightforward computation shows that $\mathrm{wt}_{2}<\mathrm{wt}_{1}$, since we have $a \leq k-1$ and $b \leq \ell-1$ (a permutation of length $m+1$ ending in an ascent can have at most $m-1$ descents). This implies that if $\pi_{L}$ has two or more components, these can be merged in the way described above, preserving the number of descents in $\pi_{L}$ while increasing its weight. Repeating that process we can therefore replace $\pi_{L}$ by a single component preserving the number of descents and increasing its weight. Thus, if $\pi$ 
has maximal weight, we can construct a permutation $\pi^{\prime}$ with maximal weight and $\operatorname{des} \pi^{\prime}=\operatorname{des} \pi$, where $\pi^{\prime}$ has a single component preceding its minimum element.

An argument similar to the one above for the components of $\pi_{L}$ can now be used to show that given $\pi^{\prime}$ we can construct a permutation $\pi^{\prime \prime}$ with the same number of descents and greater weight, whose $\pi_{L}$ is empty. More precisely, if the lengths of $\pi_{L}$ and $\pi_{R}$ are $\ell$ and $k$, respectively, and their numbers of descents are $a$ and $b$, then the maximum possible weight of $\pi_{L} \cdot 1 \cdot \pi_{R}$ is

$$
a(\ell-2-a)+b(k-1-b),
$$

whereas the maximum possible weight of a single permutation of the same length is (by the inductive hypothesis)

$$
(a+b+1)(\ell+k-a-b-1) .
$$

By the inductive hypothesis the $\pi_{R}$ of $\pi^{\prime \prime}$ must be of the form

$$
2 \ldots k n(n-1) \ldots k+1 \text {, }
$$

and together with the prepended 1 this $\pi^{\prime \prime}$ is of the form described in the statement of the theorem.

6.12. We finish by discussing some further questions about the polynomials $E_{n}(x, q)$.

First, for fixed $d$, the coefficients of $x^{d}$ in $E_{n}(x, q)$ appear to stabilize to a fixed sequence (depending on $d$ ) as $n \rightarrow \infty$. For example, for large $n$, the coefficient of $x^{2}$ becomes

$$
q^{N}+4 q^{N-1}+11 q^{N-2}+31 q^{N-3}+65 q^{N-4}+157 q^{N-5}+298 q^{N-6}+\cdots,
$$

where $N=2(n-3)$ is the maximal weight from Theorem 6.10, This (conjectural) stabilization means one can define a power series $W_{d}(t) \in \mathbb{Z} \llbracket t \rrbracket$ for each $d \geq 1$ : if for $n$ large the coefficient of $x^{d}$ stabilizes to

$$
q^{N}+a_{1} q^{N-1}+a_{2} q^{N-2}+\cdots,
$$

where now $N=d(n-1-d)$, then we put

$$
W_{d}(t)=1+a_{1} t+a_{2} t^{2}+\cdots \text {. }
$$

For example,

$$
\begin{aligned}
& W_{1}(t)=1+3 t+7 t^{2}+15 t^{3}+31 t^{4}+63 t^{5}+127 t^{6}+\cdots \\
& W_{2}(t)=1+4 t+11 t^{2}+31 t^{3}+65 t^{4}+157 t^{5}+298 t^{6}+\cdots, \\
& W_{3}(t)=1+5 t+16 t^{2}+41 t^{3}+112 t^{4}+244 t^{5}+542 t^{6}+\cdots, \\
& W_{4}(t)=1+6 t+22 t^{2}+63 t^{3}+155 t^{4}+393 t^{5}+869 t^{6}+\cdots .
\end{aligned}
$$

From this data one can make some observations. For instance, the coefficient of $t$ in $W_{d}(t)$ is clearly $d+2$, and the coefficient of $t^{2}$ is clearly $\left(d^{2}+5 d+8\right) / 2$. The coefficients also enjoy many Pascal-like relations, such as $4+7=11,5+11=16$, 
$41+22=63$. Finally, there is a connection between the coefficients of $W_{d}$ and the sequence A256193 due to Alois P. Heinz in OEIS [15]. By definition, this is the triangle $T(n, k)$ of partitions of $n$ written with two colors, where each partition contains exactly $k$ parts of the second color. For example (using a prime to denote the second color), we have

$$
\begin{aligned}
& T(3,0)=3, \text { corresponding to } 111,21,3, \\
& T(3,1)=6, \text { corresponding to } 1^{\prime} 11,11^{\prime} 1,111^{\prime}, 2^{\prime} 1,21^{\prime}, 3^{\prime}, \\
& T(3,2)=4, \text { corresponding to } 1^{\prime} 1^{\prime} 1,1^{\prime} 11^{\prime}, 11^{\prime} 1^{\prime}, 2^{\prime} 1^{\prime}, \\
& T(3,3)=1, \text { corresponding to } 1^{\prime} 1^{\prime} 1^{\prime} .
\end{aligned}
$$

A short table of the $T(n, k)$, with $k$ constant along the rows, is given below (Table 3 ). One sees that the coefficients of $W_{d}$ agree with the $T(n, k)$ up to a point, and then begin to diverge. We don't know an explanation of this connection, or why the sequences diverge.

Finally, Eulerian polynomials have been defined for all finite Coxeter groups (cf. [5]), and $q$-Eulerian polynomials have been defined for them in some cases [3]. It would be interesting to understand the proper generalization of the concepts in this paper to finite Coxeter groups.

$\begin{array}{cccccccccc}\mathbf{1} & \mathbf{3} & 6 & 12 & 20 & 35 & 54 & 86 & 128 & \ldots \\ & \mathbf{1} & \mathbf{4} & \mathbf{1 1} & 24 & 49 & 89 & 158 & 262 & \ldots \\ & & \mathbf{1} & \mathbf{5} & \mathbf{1 6} & \mathbf{4 1} & 91 & 186 & 351 & \ldots \\ & & & \mathbf{1} & \mathbf{6} & \mathbf{2 2} & \mathbf{6 3} & \mathbf{1 5 5} & 342 & \ldots\end{array}$

TABLE 3. The numbers $T(n, k)$

6.13. Remark. Using similar ideas that lead to the definition of weight for permutations, one can define a weight for complete nonambiguous trees. To do this, one notes that each internal vertex of a complete nonambiguous tree $T$ corresponds to an edge in the fully tiered tree $T^{\prime}$. If $T^{\prime}$ has weight 0 , then it means that during its construction each of its edges is being connected to the minimal vertex available. To build a higher weight tree, we connect using vertices with higher labels to increase the numbers $w_{i}$; on $T$, this can be recorded by labeling its internal vertices with the nonnegative integers $w_{i}$. The maximal such labeling gives the highest weight fully tiered tree $T^{\prime}$ that can be built from $T$, and thus defines the weight of $T$. This leads to a $q$-analogue of the numbers in the series (10), and in fact leads to another weight function on a subset of $S_{n}$, namely those permutations that do not take 1 to $n$ and $n$ to 1 (the connection is given by the positions of the leaves in the complete nonambiguous tree, or equivalently the vertex labels of the tiers in the fully tiered tree). The weight of such a permutation is then defined to be the highest weight of any 
complete nonambiguous tree that can be built from it. We have not explored these weights systematically.

\section{REFERENCES}

[1] J.-C. Aval, A. Boussicault, M. Bouvel, and M. Silimbani, Combinatorics of non-ambiguous trees, Adv. in Appl. Math. 56 (2014), 78-108.

[2] P. Boalch, Simply-laced isomonodromy systems, Publ. Math. IHES 116 (2012), no. 1, 1-68.

[3] F. Brenti, q-Eulerian polynomials arising from Coxeter groups, European J. Combin. 15 (1994), no. $5,417-441$.

[4] L. Carlitz, q-Bernoulli and Eulerian numbers, Trans. Amer. Math. Soc. 76 (1954), 332-350.

[5] A. M. Cohen, Eulerian polynomials of spherical type, Münster J. Math. 1 (2008), 1-7.

[6] S. Corteel, D. Forge, and V. Ventos, Bijections between affine hyperplane arrangements and valued graphs, European J. Combin. 50 (2015), 30-37.

[7] D. Forge, Linial arrangements and local binary search trees, ArXiv e-prints (2014), arXiv:1411.7834.

[8] I. M. Gel'fand and V. V. Serganova, Combinatorial geometries and the strata of a torus on homogeneous compact manifolds, Uspekhi Mat. Nauk 42 (1987), no. 2(254), 107-134, 287.

[9] I. M. Gel'fand, M. I. Graev, and A. Postnikov, Combinatorics of hypergeometric functions associated with positive roots, The Arnold-Gelfand mathematical seminars, Birkhäuser Boston, Boston, MA, 1997, pp. 205-221.

[10] C. Godsil and G. Royle, Algebraic graph theory, Graduate Texts in Mathematics, vol. 207, Springer-Verlag, New York, 2001.

[11] P. E. Gunnells, E. Letellier, and F. Rodriguez Villegas, Torus orbits on homogeneous varieties and Kac polynomials of quivers, Math. Zeit. (to appear).

[12] A. Postnikov, Intransitive trees, J. Combin. Theory Ser. A 79 (1997), no. 2, 360-366.

[13] A. Postnikov and R. P. Stanley, Deformations of Coxeter hyperplane arrangements, J. Combin. Theory Ser. A 91 (2000), no. 1-2, 544-597, In memory of Gian-Carlo Rota.

[14] J. Shareshian and M. L. Wachs, q-Eulerian polynomials: excedance number and major index, Electron. Res. Announc. Amer. Math. Soc. 13 (2007), 33-45 (electronic).

[15] N. J. A. Sloane, Online Encyclopedia of Integer Sequences, available at oeis.org.

[16] R. P. Stanley, Hyperplane arrangements, interval orders, and trees, Proceedings of the National Academy of Sciences 93 (1996), no. 6, 2620-2625, arXiv:http://www.pnas.org/content/93/6/2620.full.pdf.

[17] R. P. Stanley, Binomial posets, Möbius inversion, and permutation enumeration, J. Combinatorial Theory Ser. A 20 (1976), no. 3, 336-356.

[18] R. P. Stanley, Enumerative combinatorics. Vol. 2, Cambridge Studies in Advanced Mathematics, vol. 62, Cambridge University Press, Cambridge, 1999, With a foreword by Gian-Carlo Rota and appendix 1 by Sergey Fomin. 
26 WILLIAM DUGAN, SAM GLENNON, PAUL E. GUNNELLS, AND EINAR STEINGRÍMSSON

Department of Mathematics and Statistics, University of Massachusetts, Amherst, MA 01003-9305

E-mail address: wdugan@umass.edu

Department of Mathematics and Statistics, University of Massachusetts, Amherst, MA 01003-9305

E-mail address: glennon@math.umass.edu

Department of Mathematics and Statistics, University of Massachusetts, Amherst, MA 01003-9305

E-mail address: gunnells@math.umass.edu

Department of Computer and Information Sciences, University of Strathclyde, Livingstone Tower, 26 Richmond Street, Glasgow, G1 1XH, UK

E-mail address: einar@alum.mit.edu 